\title{
SUI PERIODI DI OSCILLAZIONE LIBERA DEL VERBANO
}

\section{Pietro Caloi}

J. - In un livoro precedente, dedicalo alle sesse del lago Maggiore ('), o-nertatamo the la forma della curva normale relativa al lago era co-i complicata da rendere "ardua se non impossibile " l'applicazione di uno dei metodi proposti da Chrystal. Ho voluto egualmente cimentarmi in questo sen=o, anche perché, la complessita del latgo fir si che i valori calcolati per i periodi delle sese relative siano sen-ibilmente diveri a secenda del metodo neato, specie per quanto -i riferise alle oxillazion di minor nodalità.

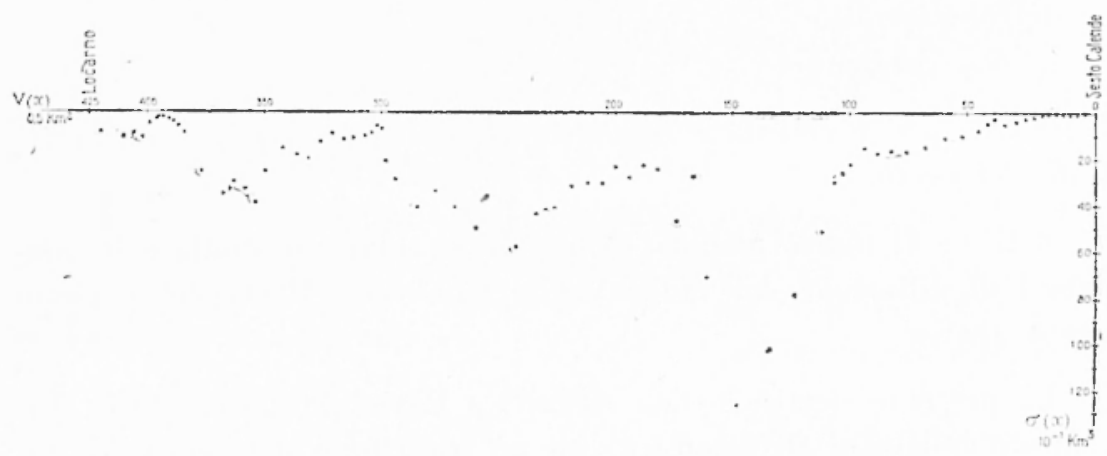

Fig. 1

Fra quelli proposi da Chry-tal, ho ritenuto che il solo metodo che schematiza la curra normale in tratti rettilinei raceordati fose applicabile, ron qualche speranza di suecesso.

Nella seconda parte di un mio lavoro sulle sesic del Garda ( $\left.{ }^{2}\right)$ ho riassunto lat relativa teoria, reltificando nna sua inceatlezza. Perlinto, mi limito qui a xiportare le equazioni che damno grli spostamenti orizzontale e rerticale

$$
\left.\begin{array}{l}
\xi u=\left\{A J_{1}(w) \div B Y_{1}(w)\right\} \sin n(t-\tau) \\
\zeta=\frac{h}{2 a}\left\{A J_{0}(w)+B Y_{0}(w)\right\} \sin n(t-\tau),
\end{array}\right\}
$$


Ane a. h sono rostanti legatle dallat relazione $h(x)=h\left(1-\frac{x}{a}\right.$, rela-

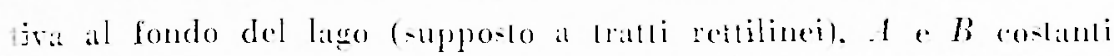

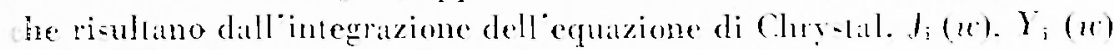
- $i=0.1$ - funzioni di Besed e Teumam dell arsomento

$$
u-2 n a|\overline{1-x a}| \bar{g} h
$$

ando $n=\frac{2 \pi}{T}(\Gamma=$ periodo $)$.

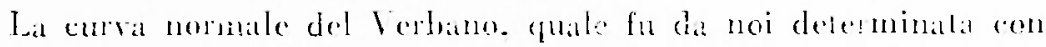

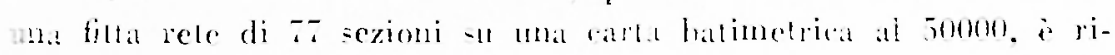
proctolta nella fị. 1 .

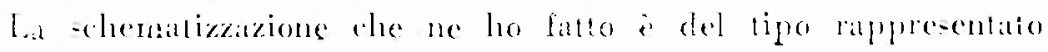
nella fig. 2. Poniamo

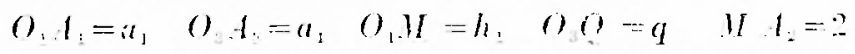

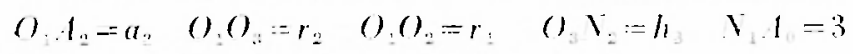

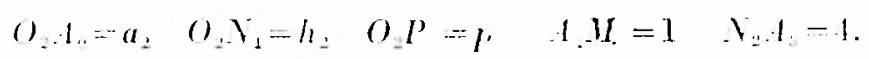

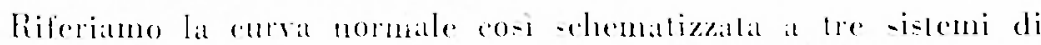

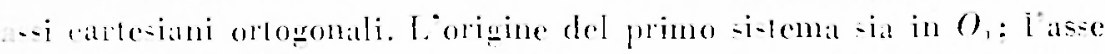

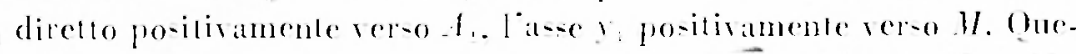

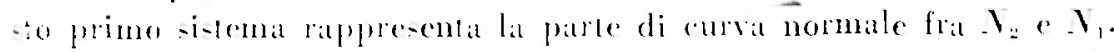
Sia O. l'origine del secondo -i-lemil ratle-iano. Le coordinale di $O$

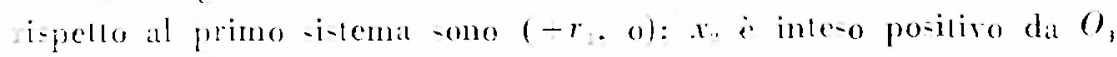

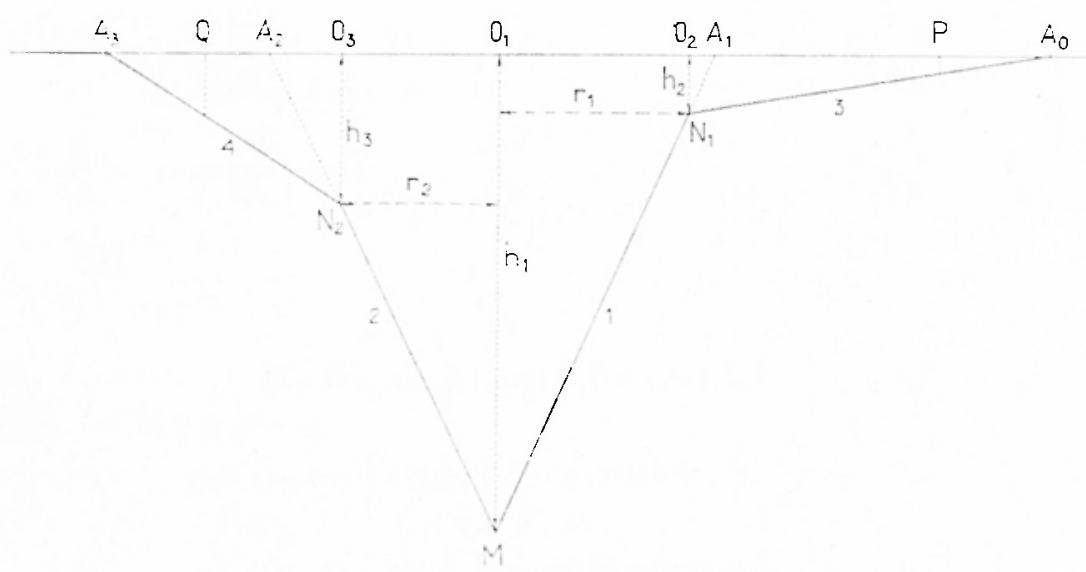

Fig. 2 
rerso O.. ye posilivo da $O$., verso $Y_{1}$. II terzo sistema ha orivine in $O:, x_{3}$ esendo positivo contato verso $O_{1}, y_{3}$ positivo da $O_{3}$ rerso $X_{\text {. }}$.

Le coordinate di $O_{3}$ rispello al primo sistema sono $\left(-r_{.}, 0 \%\right.$ lireo le erpuzioni delle relle l e 2, riferile al primo sistema:

$$
h_{1}(x)=h_{1}\left(1-\frac{x}{a_{1}}\right), h_{2}(x)=h_{1}\left(1+\frac{x}{a_{: 3}}\right) .
$$

Lianalogal dellat 3 riferita al secondo sistema

$$
h_{1.1}(x)=h_{22}\left(1-\frac{x}{a_{2}}\right)
$$

4 quella dellat fiferila al ter\%o iselema

$$
h_{4}(x)=h_{4 .}\left(1+\frac{x}{a_{4}}\right)
$$

Vengono inoltre le relazioni

$$
a_{i}=-r \frac{h_{1}}{h_{1}-h_{*}} \quad ; \quad a_{: i}=r_{:} \frac{h_{1}}{h_{1}-\frac{h_{i ;}}{2}} .
$$

Poniamo, ricordando la [2],

$$
\begin{aligned}
& \left\{\begin{array}{l}
w_{1}=\frac{2 n a_{1}}{1 g h_{1}} \sqrt{1-\frac{x}{a_{1}}} ; \quad u_{1}=\frac{2 a_{1}}{1 g h_{1}} \quad ; \quad \beta_{1}=\frac{2 a_{1}}{1 g h_{1}} \sqrt{1-\frac{r_{1}}{a_{1}}} \\
w_{2}=\frac{2 n a_{2}}{1 g h_{2}} \sqrt{1-\frac{x}{a_{2}}} \quad ; \quad u_{3}=\frac{2 u_{2}}{1 g h_{2}} \quad ; \quad \theta_{2}=\frac{2 a_{2}}{1 g h_{2}} \sqrt{1-\frac{P}{a_{2}}}
\end{array}\right.
\end{aligned}
$$

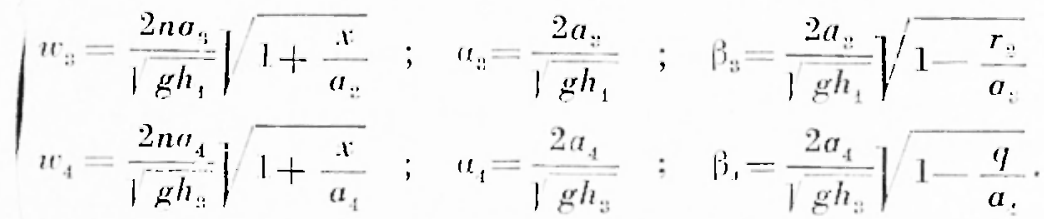

les le [1] avremo

$$
\begin{aligned}
& \Xi_{1}^{2} w_{1}=\left\{A_{1} I_{1}\left(w_{1}\right)+B_{1} Y_{1}\left(w_{1}\right)\right\} \sin n(1-\tau) \\
& \therefore=\frac{h_{1}}{2 a_{1}}-\left\{A_{1} J_{1}\left(u_{1}\right)+B_{1} Y_{n}\left(u_{1}\right)\right\} \sin n(l-T) \\
& w_{2}=\left\{I_{2} J_{1}\left(w_{2}\right)+B_{2} Y_{1}\left(u_{2}\right)\right\}_{j} \sin n(1-\tau)
\end{aligned}
$$




$$
\begin{aligned}
& \left.\because=\frac{h_{0}}{2} u_{:} A_{2} J_{0}\left(u_{2}\right)+B_{2} Y_{n}\left(u_{2}\right)\right\}_{j}=\text { in } n(t-\tau) \\
& \xi_{3}, u_{3}=\left\{A_{3} J_{1}\left(u_{3}\right)+B_{3} Y_{1}\left(w_{3}\right)\right\}_{i}=\operatorname{inn} n(l-\tau)
\end{aligned}
$$

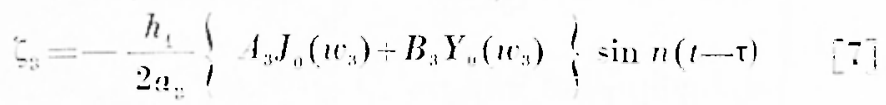

$$
\begin{aligned}
& \xi_{4} w_{4}=\left\{A_{1} J_{1}\left(w_{4}\right)+B_{1} I_{1}\left(w_{1}\right)\right\} \text { in } n(1-\tau) \\
& \left.c_{4}=-\frac{h_{n}}{2} ; A_{1} J_{n}\left(w_{4}\right) \div B_{1} Y_{0}\left(w_{4}\right)\right\}_{i} \sin n(t-\tau)
\end{aligned}
$$

dove le prime due. la $5^{\mathrm{h}}$ e la $6^{\mathrm{a}}$ sono riferite al primo si=lema, mume tre la $3^{a}$ e la $f^{:}$-ono riferile al seconde sistena e le ultime due at terzo sistema.

Velle $[\because]$ sono da determinare le nove costinti $A_{1}, B_{1}, A, B \ldots$ $B_{1}, n$, valendoed delle condizioni ai limiti. Consideriamo delle prim: otto il rapporto di/Bi. Te condixioni ai limiti sono:

agli estremi $P$ e Q del ligo derenere $\equiv=0$ :

Inoline. per.

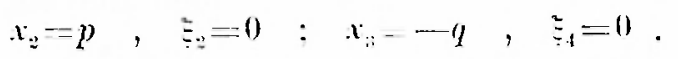

Per il punlo 0..

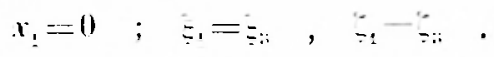

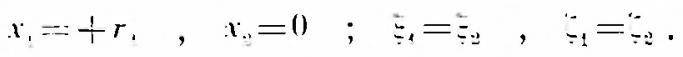

Per ii punto 0

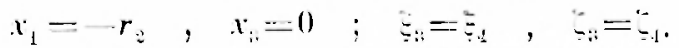

Dille [7]. ricordando le 6 ] e per le [8], si hal

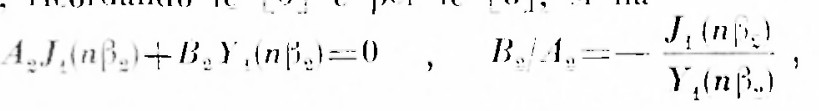

oppure

$$
D_{1} A_{4}=-\frac{J_{1}\left(n\left(i_{1}\right)\right.}{I_{1}\left(n \beta_{4}\right)}
$$

Per $x_{1}=0+\frac{u_{1}}{u_{i}}=\frac{a_{1}}{a_{2}} ;$ per $x_{1}=+r_{1}, x_{2}=0$ i ha $w_{1} / w_{2}-y_{1} u_{0}$;

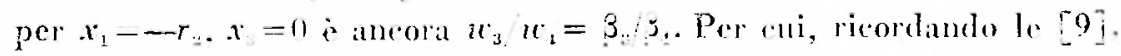
[10] e [11] e poso

$$
\begin{aligned}
& P_{1}\left(P_{2}, n_{0}\right)=Y_{1}\left(n P_{0}\right) J_{1}\left(n u_{0}\right)-J_{1}\left(n+P_{2}\right) Y_{1}\left(n u_{0}\right) \\
& P_{0}\left(\beta_{3},\left(c_{0}\right)=Y_{1}\left(n \beta_{2}\right) J_{0}\left(n\left(t_{0}\right)-J_{1}\left(n \beta_{z}\right) Y_{11}\left(n \alpha_{2}\right)\right.\right. \\
& P_{1}\left(\beta_{1},{ }_{1}\right)=X_{1}\left(n \beta_{1}\right) J_{1}\left(n u_{1}\right)-J_{1}\left(n \beta_{1}\right) Y_{1}\left(n u_{1}\right) \\
& P_{0}\left(P_{1},\left(u_{4}\right)=Y_{1}\left(n P_{1}\right) J_{0}\left(n u_{1}\right)-J_{1}\left(n P_{4}\right) Y_{0}\left(n u_{4}\right)\right. \text {, }
\end{aligned}
$$


-i hil

$$
\begin{aligned}
& I_{1} J_{1}\left(n u_{1}\right)-B_{1} Y_{1}\left(n+u_{1}\right)=\frac{a_{1}}{a_{0}}\left[A_{3} J_{1}\left(n u_{0}\right): B . Y .\left(n u_{0}\right)\right]
\end{aligned}
$$

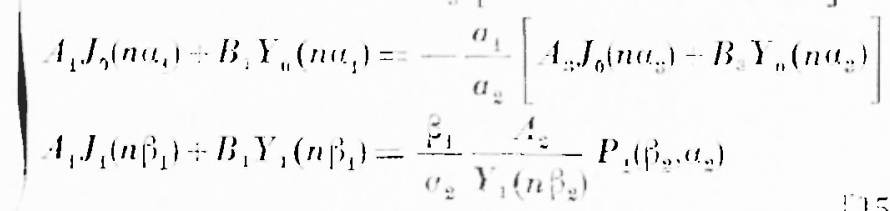

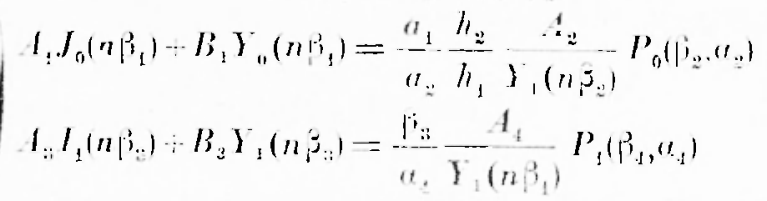

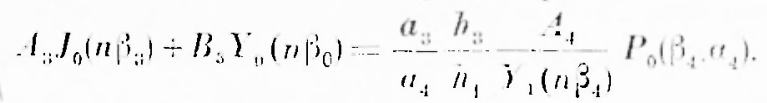

Il deleminante dei coeffieienti delle ineonnite $A_{1}, B_{1}, A_{2}, A_{3}$, B. A: uguagliato a zero, da l'equazione dei periodi:

$$
\begin{aligned}
& J_{1}\left(n a_{1}\right) \quad Y_{1}\left(n a_{1}\right) \quad 0 \quad-\frac{a_{1}}{a_{3}} J_{1}\left(n u_{3}\right) \\
& J_{0}\left(n u_{1}\right) \quad Y_{0}\left(n r_{1}\right) \quad 0 \quad \frac{u_{1}}{a_{3}} J_{0}\left(n u_{0}\right) \\
& J_{1}\left(n \beta_{1}\right) \quad Y_{1}\left(n \beta_{1}\right)-\frac{P_{1}}{{ }_{2}} \frac{P_{1}\left(\rho_{2} \mu_{2}\right)}{Y_{2}\left(n \beta_{2}\right)} \quad 0 \\
& J_{0}\left(n P_{1}\right) \quad Y_{0}\left(n \gamma_{1}\right)-\frac{a_{1} h_{2}}{a_{2} h_{1} P_{0}\left(P_{2},\left(n_{2}\right)\right.} \quad 0 \\
& \text { () } 1) \quad J_{i}\left(n \beta_{0}\right) \\
& \text { (1) } 11 \quad J_{0}\left(t_{0},:\right)
\end{aligned}
$$

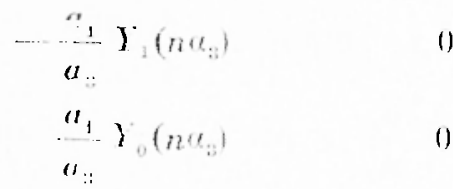

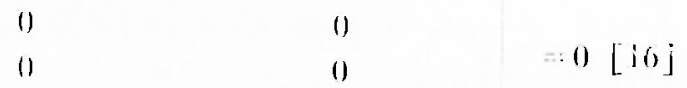

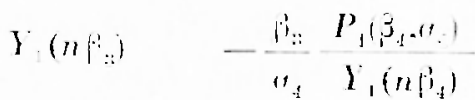

$$
\begin{aligned}
& Y\left(n, j_{3}\right) \quad-\frac{a_{3}}{a_{4}} \frac{h_{3}}{h_{1}} \frac{P_{0}\left(\beta_{4}, a_{4}\right)}{Y_{i}\left(n \beta_{4}\right)}
\end{aligned}
$$


Ia [16] vale nell”jpotesi che onli estremi del lago siano smus-ati In realti, in $f_{11}, A_{3}$ il latgo termina ad angolo acuto. Considerand. questo come un caso limite della precedente rappresentazione. po.. siamo porre $p=a_{1}, q=a_{1}$. Nelle [4], [5] arremo pertanto

E' inolue

$$
\beta_{2}=0, \beta_{1}=0 \text {. }
$$

$$
\lim _{\beta_{2}=0} \frac{I_{1}\left(n \beta_{2}\right)}{Y_{1}\left(n \beta_{2}\right)}=0: \quad \lim \frac{J_{1}}{\beta_{1}=0} \frac{\left(n_{\beta_{1}}\right)}{Y_{1}\left(n \beta_{1}\right)}=0 ;
$$

per cui dalle $[1+]$

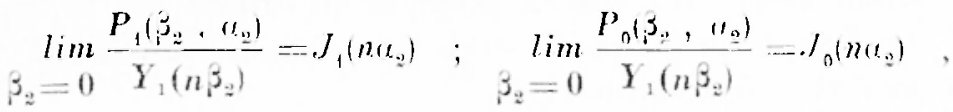

$$
\begin{aligned}
& \lim _{\beta_{1}=0} \frac{p_{1}\left(\beta_{1}, \alpha_{1}\right)}{Y_{1}\left(n_{3}\right)}=J_{1}\left(n u_{1}\right) \quad ; \quad \lim _{1} \frac{P_{1}\left(\beta_{4}, \alpha_{2}\right)}{Y_{1}\left(n_{1}\right)}=J_{0}\left(n u_{1}\right)
\end{aligned}
$$

La $\lfloor 16\rceil$, osiervando che

$$
\frac{\beta_{1}}{a_{0}}=\frac{a_{1}}{a_{0}} \frac{h_{:}}{h_{1}} \quad ; \quad \frac{j_{*}}{a_{i}}=\frac{a_{:}}{a_{4}} \frac{h_{:}}{h_{1}},
$$

diviene allora, moltipliamdo !a $5^{a}$ e la $\sigma^{2}$ riga per $\frac{a_{0}}{a_{*}}$,

$$
\begin{aligned}
& J_{1}\left(n u_{1}\right) \quad Y_{1}\left(n u_{1}\right) \quad 0 \quad-J_{1}\left(n u_{1}\right)-Y_{1}\left(n u_{3}\right) \quad 0 \\
& J_{0}\left(n \alpha_{0}\right) \quad Y_{n}\left(n u_{1}\right) \quad 0 \quad I_{0}\left(n x_{0}\right) \quad Y_{0}\left(n u_{0}\right) \quad 0
\end{aligned}
$$

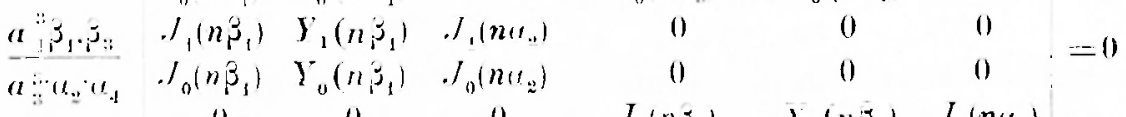

$$
\begin{aligned}
& \begin{array}{llllll}
0 & 0 & 0 & J_{1}\left(n \beta_{i:}\right) & J_{1}\left(n \beta_{: !}\right) & J_{1}\left(n u_{1}\right)
\end{array} \\
& 00 \quad 0 \quad J_{0}\left(n \beta_{0}\right) \quad I_{n}\left(n \beta_{0}\right) \quad J_{0}\left(n u_{1}\right)
\end{aligned}
$$

2. - Pasciamo alla soluzione dellat [17].

Dati dati relativi alla curva normale, per i quali rimando al la. voro citato ( $\left.{ }^{1}\right)$, si ottiene, nel caso della shematizazione preseelta,

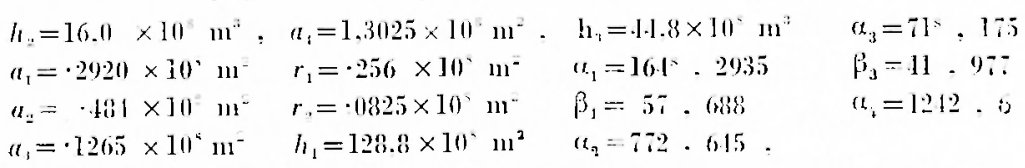

$$
\begin{aligned}
& -\frac{j_{1}}{a_{2}}=.07+606 \\
& \frac{a_{1}}{a_{3}}=2.3083 \\
& \frac{\beta_{3}}{a_{2}}=.03378
\end{aligned}
$$


I. Equarione dei periodi diviene pertanto $I)(n)$

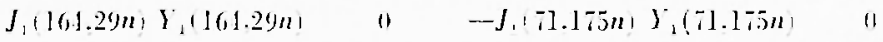

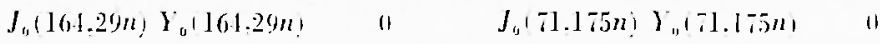

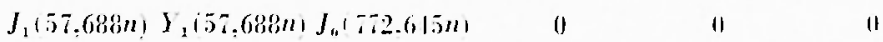

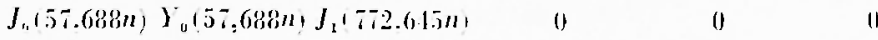

$$
\begin{aligned}
& \text { 0 } 11 \quad \| \quad J_{1}\left(11.97 \pi n Y_{1}(11.97-\pi n) J_{1} 12.12 .6 n !\right. \\
& \text { 1) } 0 \quad 0 \quad J_{0}(11.977 n) Y_{4}(11.977 n) J_{0}(1212 .(n)
\end{aligned}
$$

La ri-oluzione della [18] is stala proposa all'I-ituto per le Applirizioni del Calcolo, tanto benemerito per lausilio ralidi-simo che icmini-e alla ricerea seientifiea.

Furono desmti per le prime sei radiei positive di $D(n)$ i se-ueni valori

$$
\begin{array}{ll}
n_{1}=0.00231, & n_{1}=0.006082 \\
n_{0}=0.0035 .2 & n_{5}=0.0017 .6 \\
n_{: 3}=0.00505 & n_{1}=0.0096 .3 .
\end{array}
$$

rui corri-pondono. a meno di 5 , i periodi

\begin{tabular}{|c|c|c|c|c|}
\hline & & $T_{1}$ & $T_{z}$ & $T_{::}$ \\
\hline .ol metodo & Defant & $48^{\mathrm{m}}, 7$ & $37^{m} \cdot 4$ & $23^{m}, 4$ \\
\hline ” & Hidakal & $-4^{-\mathrm{m}} \cdot 4$ & $33^{m}, \bar{\imath}$ & $27^{m} .0$ \\
\hline
\end{tabular}

$$
\begin{array}{lll}
T_{1}=45^{m}, 35 & T_{3}=20^{m}, 75 & T=13^{m} .5 \\
T_{2}=29^{m, 75} & T_{4}=15^{12}, 35 & T_{4}=10^{m} .85
\end{array}
$$

per le prime aei sesse del Verbano.

3. - Vel lavolo precelente sulle sese del lago Magwiore i sono onenuti i seguenti valori per le sesse uni-bi- e trinodali:

Lavcordo tra $i$ valori ollenuli con $i$ tre metodi, soddisfacente jur la sesa uninodale, cessa di eserlo per la binodale, mentre è Buonis-imo per la trinodale. Queste dirersià. particolamente sensibili per la binodale, vamo attribuite alle notevoli variazioni che il harino del Jago preconta in largliezza e nell andamento della linea di valle: variazioni the lo discostano sensibilmente dalle ipoten su ui pogriano le varie teorie. Commugue, e interesante osacrare che, juerindendo dalla binodale. i periodi delle sesse minodale o trinodale calcolati ron tre metodi differizeono molto poro e la differenza $\therefore$ particolarmente piecola per la trinodale. per la quale $i$ valori otsemuti con i metodi di ITidaka e di Chrystal prationmente coincidono. 
$E^{*}$ noto che, qualunque sia il metodo usilo, i valori calcolati per i periodi delle sese di matgiore nodaliat differiotono sempre meno col cresere della nodalití. Pertanto i valori dei periodi delle sesas a ruattro, cincfue e sei nodi calcolati in questo lavoro. posono rileneri molıo prossimi a ruelli reali.

Ancora non is stato posibile oltenere huone regielazioni delle $\therefore$ ese del Verbano. Comungue dai valori finota owersati. sembra whe i periodi fornili dal melodo di Millaka siano i più allendibili.

Roma - Istituto Nazionale di Ceofisica - aprile 1918.

\section{RIASSUNTO}

P'artendo dalle basi della teoria di Cibrystal, si stiluppa un metado analitico per la determinazione dei periodi di oscillasione libera delle acque del Lago Maggiore. Risolta lequasione dei periodi costituita da un determinan!e di sesto ordine - si sono calcolati $i$ ralori dei periodi corrispondenti alle oscillazioni libere di uno. due, tre. ... sei nodi.

\section{BIBLIOGRAFIA}

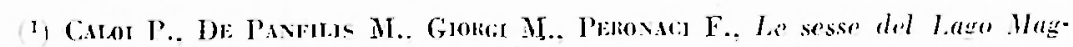
sierre. "Anmali di Gcofisica ». I, 2 (1948).

(-) C.roo P. Le sesse del Lago di Garda. Parle II a Ann. di Geol. ) I. 2 (1948). 